\title{
Status of LAGO at mount Chacaltaya
}

\author{
R. Condori ${ }^{a}$, M. Andrade ${ }^{b}$, R. Guzmán ${ }^{b}$, C. Nina ${ }^{a}$, P. Miranda ${ }^{a}$, M. Raljević ${ }^{a}$, \\ H. Rivera ${ }^{a}$, M. Subieta ${ }^{a}$ and R. Ticona ${ }^{a}$ \\ for the LAGO Collaboration \\ (http://lagoproject.org/colab.html) \\ ${ }^{a}$ Universidad Mayor de San Andrés, Bolivia \\ ${ }^{b}$ Universidad Mayor de San Simón, Bolivia
}

The Latin American Giant Observatory (LAGO) is an international experiment with multiple scientific objectives. These objectives include the study of the extreme universe, space weather and atmospheric radiation at ground level. The observatory is composed of several experimental sites, distributed in the American continent, from Argentina to Mexico. The highest LAGO site is located on the Chacaltaya mountain, Bolivia, at an altitude of nearly 5300 m.a.s.l. In this document we report the status of the experimental site on Chacaltaya. In particular, emphasis is given on the development of a water Cherenkov detector. We describe the pilot detector that we are assembling, the energy calibration method and the characterization of the PMT employed.

35th International Cosmic Ray Conference - ICRC2017

10-20 July, 2017

Bexco, Busan, Korea 


\section{Introduction}

The laboratory located on mount Chacaltaya is the highest and oldest scientific facility in continuous operation in the world. Its origins can be traced back to the installation of a meteorological station, belonging to the Bolivian Air Force, by Ismael Escobar Vallejo in September 1942. A few years later, using nuclear emulsion plates on Chacaltaya, Lattes, Occhialini, Powell and their collaborators discovered the pion [1]. Encouraged by this remarkable finding, Escobar presented a proposal to the Universidad Mayor de San Andrés for the construction of a laboratory on Chacaltaya, which was immediately accepted. The laboratory officially began its activities on January 1st, 1952, having Escobar as its first director, and since then has hosted various experiments, mainly in the areas of cosmic ray physics and atmospheric physics. For further historical information the reader is referred to [2].

The laboratory on Chacaltaya is located at $16^{\circ} 21^{\prime} 0.5^{\prime \prime} \mathrm{S}$ latitude and $68^{\circ} 7^{\prime} 53.1^{\prime \prime} \mathrm{W}$ longitude. The geomagnetic rigidity cutoff at the site is $12.5 \mathrm{GV}$ and its elevation is 5260 m.a.s.l. The atmospheric depth is $530 \mathrm{~g} / \mathrm{cm}^{2}$, corresponding to a mass burden of roughly 14 radiation lengths.

An advantage of having a high-altitude site for time series recording of secondary particles at ground level is the increased sensitivity with respect to lower altitude sites. Given that Gamma Ray Bursts (GRB) and space weather monitoring are generally carried out by analyzing temporal count variations induced on different types of detectors, this advantage is a fundamental one for the scientific purposes of LAGO. As an example of the potential offered by high-altitude sites, figure 1 shows lines of equal sensitivity for experiments of different elevation and size [3]. The plot was produced to illustrate the sensitivity of the "single particle technique" [4], originally intended to identify increases in the background rate of a detector exposed to secondary particles in the atmosphere, due to an excess of lonely particles, survivors of shallow showers generated by the massive arrival of gamma rays on the upper atmosphere.

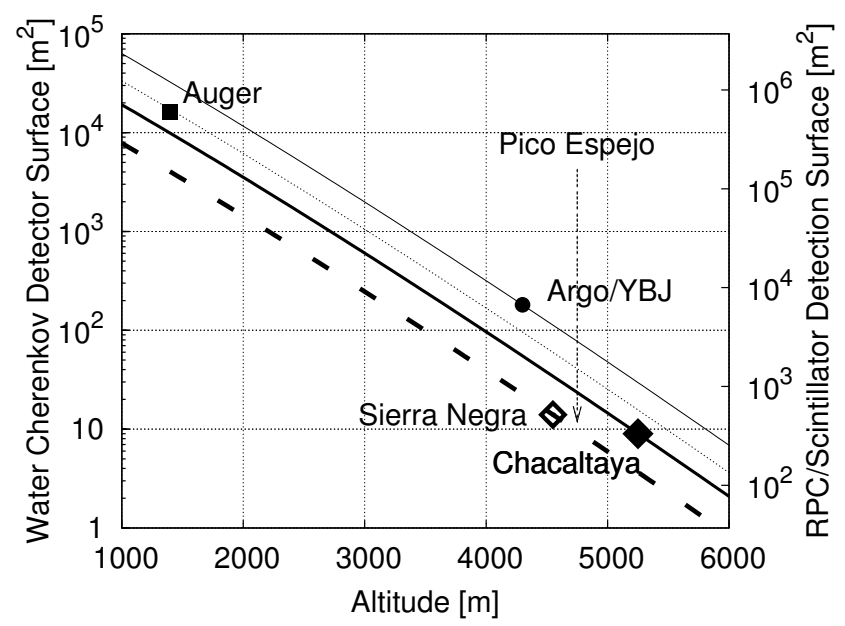

Figure 1: Sensitivity of the single particle technique for water Cherenkov detectors (WCD), resistive plate chambers (RPC) and scintillators as a function of altitude, according to simulations. The area necessary to achieve the same sensitivity is noted on the ordinate. The Argo Yangbaijing (YBJ) experiment (light solid line) and the Pierre Auger Observatory (medium solid line) are shown together with two LAGO sites, Chacaltaya (thick solid line) and Sierra Negra (dashed line). Figure extracted from [3]. 
Three water Cherenkov detectors (WCD) had been running intermittently on Chacaltaya, from 2009 to 2014, as part of LAGO. Nonetheless, a number of issues caused their stoppage in 2014, with the intention of replacing those detectors with new ones with better characteristics. In this document, we report the status of the ongoing work.

\section{The water Cherenkov detector}

We are developing a pilot WCD at the Universidad Mayor de San Andrés for a future implementation in the laboratory at Chacaltaya. Our research installations are located in the city of La Paz, Bolivia, at 3450 m.a.s.l., at the university campus of Cota Cota.

The detector itself is a cylindric plastic tank, whose external surface has been covered with sheets of asphalt membrane in order to minimize light leakages from the outside to the inside. The tank dimensions are $87 \mathrm{~cm}$ of diameter and $100 \mathrm{~cm}$ of height. The detector is filled with pure water up to $78 \mathrm{~cm}$, which we distill so as to increase the absorption length of Cherenkov photons. We measured the resistivity of water after the distillation process and it is around $1 \mathrm{M} \Omega \cdot \mathrm{cm}$. This is a gain with respect to the WCDs previously operating on Chacaltaya, and has the potential of improving to a large degree the ability of the WCD to perform measurements of energy deposited in its volume. We will further explore this possibility in the future. Figure 2 shows a photograph of the WCD prototype assembled in our laboratory at Cota Cota.

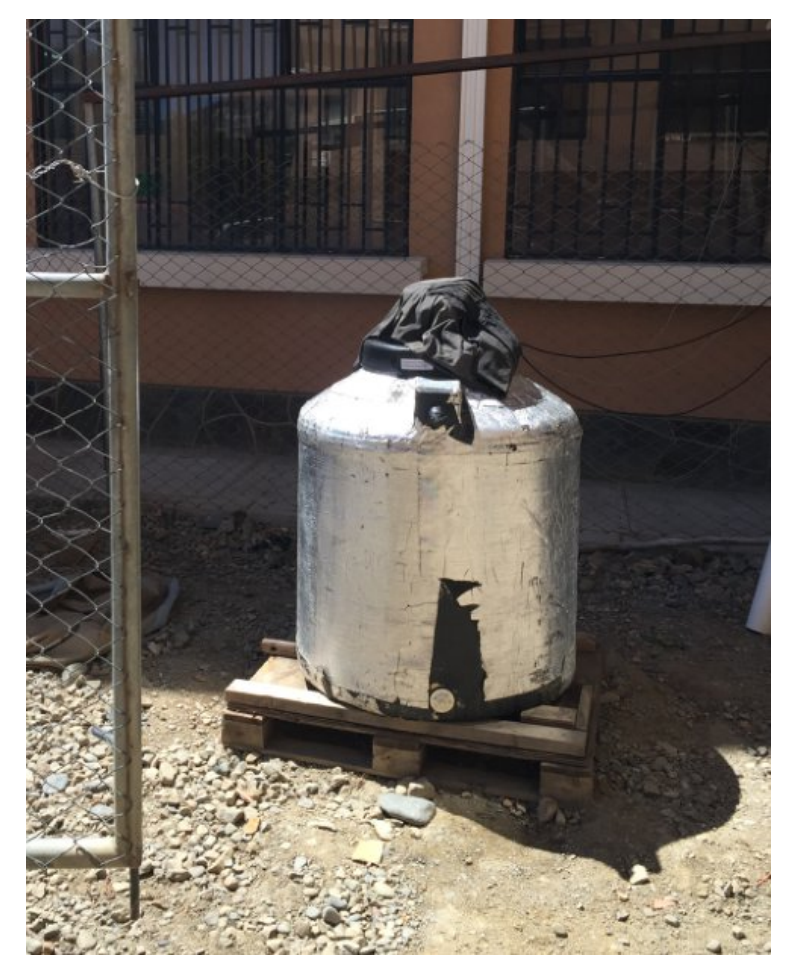

Figure 2: The prototype WCD situated at the university campus in Cota Cota. The PMT case can be seen at the top of the tank.

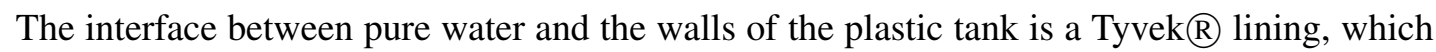
is commonly used in WCDs because of its high diffuse reflectance $[5,6,7]$. The body of water is 
observed from above by an 8 inch photomultiplier tube (PMT) made by THORN EMI, model 9350 $\mathrm{KA}$, which we placed at the center of the tank.

We measured the charge spectrum for dark pulses produced in the photomultiplier operating at $1320 \mathrm{~V}$. The resulting histogram is shown in figure 3. Part of the pulses recorded can be attributed to single electrons, spontaneously emitted by the photocathode through thermionic emission. These electrons arrive to the first dynode and start the current multiplication process, and are thus capable of emulating the signal produced by a single photon. The signals generated by single electrons show as a hump around $20 \mathrm{pC}$ in the distribution.

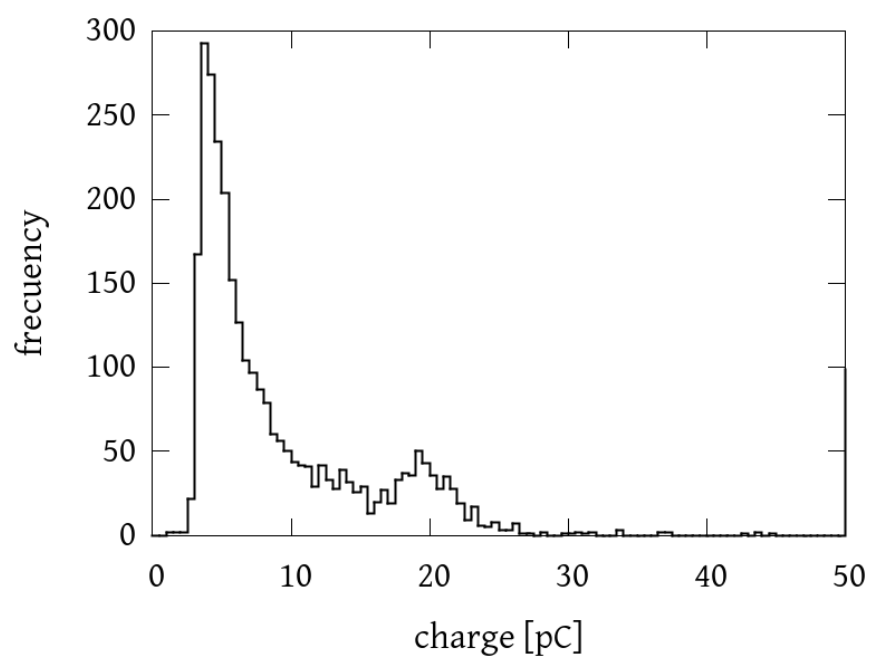

Figure 3: Charge histogram obtained for dark counts in the PMT used for the prototype WCD. The hump around $20 \mathrm{pC}$ is caused by single electrons spontaneously produced at the photocathode by thermionic emission.

As a first step to characterize the detector as a whole, we assembled a two-fold coincidence system to extract signals from the WCD, produced by vertical muons that cross the detector by its geometrical axis. We used two polyvinyltoluene (PVT) scintillators $(25.5 \mathrm{~cm} \times 25.5 \mathrm{~cm} \times$ $0.8 \mathrm{~cm}$ ) which we placed over and under the WCD, spaced by a distance of roughly $2.5 \mathrm{~m}$. The temporal coincidence of signals coming from these two scintillators form the trigger condition that we require to extract a signal from the WCD and attribute it to the passage of a vertical muon. The plot in figure 4 shows a typical event recorded by the three-detector system.

The distribution of the total charge collected for vertical muons in the WCD is shown in figure 5 (the so-called vertical equivalent muon, or VEM). At a first glance, it qualitatively resembles the distribution expected for energy loss straggling due to ionization in thin absorbers [8]. This distribution can be used to calibrate in energy the signals produced by a WCD.

As a final remark, it is interesting to note that the mean value for VEM signals is $2.18 \pm 0.05 \mathrm{nC}$, which approximately corresponds to 110 photoelectrons arriving to the first dynode of the PMT. This result is in good agreement with what has previously been found using Monte Carlo simulations of a WCD [9]. 


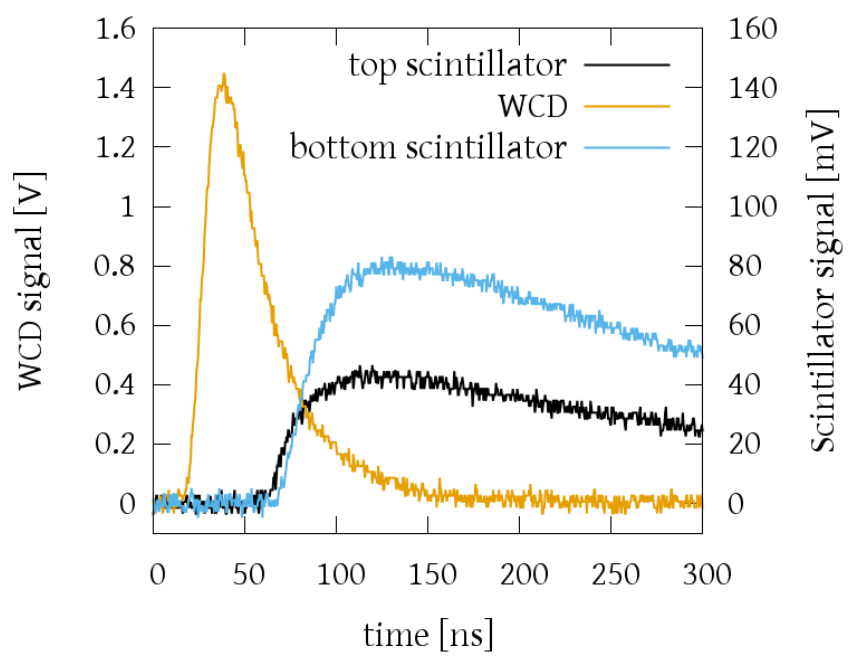

Figure 4: Signal recorded by the WCD, together with signals from two scintillators, in response to the passage of a vertical muon that interacts with the set of detectors. The time of flight measured by both scintillators (approximately $8 \mathrm{~ns}$ ) corresponds to $\sim 2.5 \mathrm{~m}$, which is the distance that separates them.

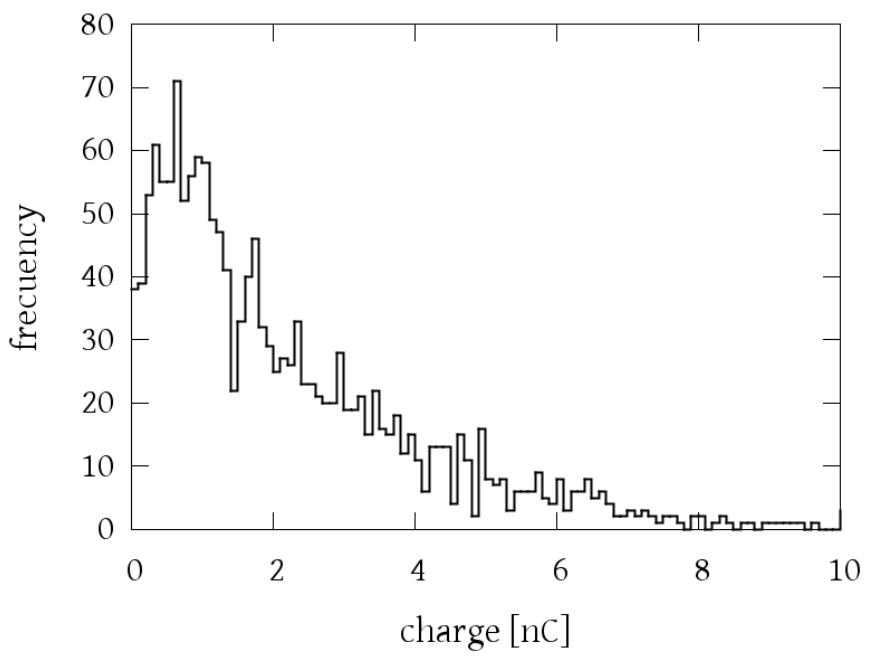

Figure 5: The charge histogram for the prototype WCD for vertical equivalent muons (VEM).

\section{Acknowledgments}

We are very thankful to the Laboratorio de Física de la Atmósfera (LFA) from the Universidad Mayor de San Andrés for lending electronic equipment to carry out some of this work.

\section{References}

[1] Lattes, C. M. G., et al., Nature, 159, 694 (1947).

[2] Aguirre, C., Medio siglo de Ciencia en Bolivia, La Paz, Fundación Universal Simón I. Patiño (1996).

[3] Bertou, X., et al., Proceedings of the 31st ICRC, Łódź, arXiv: 0906.0816v1 [astro-ph.HE], (2009). 
[4] Aglietta, M., et al., Astrophys. J., 469, 305 (1996).

[5] Gichaba, J. O., FNAL Technical Report, 15017207 (1998).

[6] Filevich, A.., et al., NIM A, 423, 108 (1999).

[7] Arteaga-Velázquez, J. C., et al., NIM A, 553, 312 (2005).

[8] Leo, W. R., Techniques for Nuclear and Particle Physics Experiments: A How-to Approach, Springer, 2nd edition (1994).

[9] Rivera Bretel, H. M., Implementación y calibración de un prototipo de detector Cherenkov de chubascos atmosféricos, Bachelor's thesis, Universidad Mayor de San Andrés (2007). 